\title{
A GENERIC FRAMEWORK BASED ON MACHINE LEARNING TECHNIQUES FOR VIRTUAL ORGANIZATION MANAGEMENT
}

\author{
Leandro Loss, Ricardo J. Rabelo, Alexandra A. Pereira-Klen \\ Federal University of Santa Catarina, Department of Automation and Systems \\ GSIGMA - Intelligent Manufacturing Systems Group \\ Florianópolis (SC),Brazil \\ \{loss, klen\}@gsigma.ufsc.br \\ rabelo@das.ufsc.br
}

\begin{abstract}
The management of Virtual Organization (VO) brings some challenges. One of them is the appropriate and effective coordination and monitoring of distributed businesses processes. It is not easy or trivial to handle the enormous amount of information about and from VOs members. As a direct consequence, decisions are crucial and should be taken not only with agility, but also with intelligence. This paper introduces a framework to support an integrated decision-making environment through which managers will be able to take assisted and smarter decisions. The key element of this framework is the Intelligent Decision Support System, which applies Artificial Intelligence techniques (Data Mining and Machine Learning) to facilitate and to support the decision-making process.
\end{abstract}

\section{INTRODUCTION}

Enterprises are even more increasing their efforts in order to improve their (intra and inter) business processes, so that they can increase their own performance and at the same time become more competitive. In other words, this means that enterprises are exposed to a very competitive, demanding and global market. Some actions associated to this "movement towards competitiveness improvement" include: implementation of advanced information technology systems, reengineering task forces, e-commerce sites, integration of legacy systems, more powerful communication infrastructures, among others. On the one hand, all these actions provided to the companies a base for receiving and sending plenty of information. On the other hand, the more the companies use information the harder is the task to treat it in an efficient and smart way. According to Rabelo \& Pereira-Klen (2002) managers have been plunged into so much information that, in opposite to the core objective, more difficulties have been brought for taking smart and agile decisions.

In this world scenario companies are being forced, due to the high level of competition, to take decisions not only with agility (an emergent requirement), but 
also with intelligence. Regarding the sort of problems that usually take place in the daily business management, it is observed that most of them are solved - when solved! - via ad-hoc actions. Actually, most of the available systems have been designed to be passive, don't offering any comprehensive supporting methodologies and/or systems guidance for decision-making. They use to be purely reactive, without considering neither the amount of knowledge the company already has about itself nor allowing the knowledge storing accordingly. Even the historical information about how decisions are taken and their related consequences is rarely stored. This means that systems are not yet enough prepared to help managers properly, regarding the issues that are indeed required in modern businesses, such as in alliances like Virtual Organizations (VOs) ${ }^{1}$ (Rabelo, 2004b). However, despite that nowadays working collaboratively is fundamental to accomplish goals and to conquer new markets, the management of such alliances is of extreme importance but at the same time much more complex than "traditional" management.

This paper introduces a system architecture that aim to offer an integrated decision-making environment through which managers will be able to take smarter decisions based on the current data of VO members and on their past decisions/actions. Artificial Intelligence (AI) techniques properly combined serve as the basis to facilitate and to support decision-making process.

The paper is organized as follows: Section 2 presents a literature review related to the approached area. Section 3 briefly depicts the basic concepts of the AI concepts and techniques used. In section 4 the proposed system architecture is introduced and framed on an industrial scenario view. Finally section 5 provides preliminary conclusions and future steps.

\section{LITERATURE REVIEW}

According to Dobler \& Burt (1996) the competition is (or should be) between value chains - not between members of a value chain. All efforts should be combined to motivate buyers and sellers to work in a collaborative mode where the forming and nurturing of buyer-seller partnerships and strategic alliances rise as a consequence. The key characteristics of such relationships are related to the interests' compatibility, mutual need, and willingness to be open sharing information as well as to the benefits resulting from the relationship and, perhaps of greatest importance, to trust $^{2}$ (Dobler \& Burt, 1996).

Considering the partnerships and the management of strategic alliances, decision-making processes are crucial in order to achieve success. However, as stated by Dobler \& Burt (1996), managers usually take decisions mostly based on "self-intuition", mental process, following a range of steps with no support tool. It makes this process, almost always, a blend of rational and emotional factors.

\footnotetext{
${ }^{1} \mathrm{~A}$ VO constitutes a number of cooperating independent organizations with certain common purposes that provides a set of services and functionalities to the outside world, as if together they represent one organization (Afsarmanesh, 2004).

${ }^{2}$ Trust is usually based on knowing the risk level in a business. Trust may speed up the interenterprise processes and decrease monitoring and coordination costs (ECOLEAD, 2005).
} 
Several research projects have studied the Virtual Organization area. Usually the focus was on issues supporting creation rather than on management actions (ECOLEAD, 2005).

VO management refers to change how single enterprises view their way of doing business. Those enterprises must transform themselves from static and hierarchical to (semi-)dynamic and flexible network of organizations (Afsarmanesh, 2004). These networks must enable the involved organizations to develop the capability of setting up their needed processes in reasonable time in order to capture business opportunities, which no single company can do on its own (Afsarmanesh, 2004).

Relevant aspects concerning VO coordination were considered in previous works which aimed to describe the coordination issues. For example, Bremer (Bremer, 1999) argued that in order to achieve a successful partnership, a set of coordination aspects may be established, such as marketing strategy and game simulation. The research was performed using simulation which allowed the core competences description to each enterprise and visualized possible business opportunities when they rose up.

Actually, the complexity of managing VOs requires several managerial actions, some depends on the manager's skills, experience and capabilities, and others do not. It is believed that if those actions were analyzed and supported by an "adviser", like smart software tool, they will be much better.

The few approaches dealing with high level management miss smart procedures and/or functions to assist different kinds of VOs which are constantly changing over time. The duty to anticipate faults or unwished actions as well as the use of lessons learnt from experience or from available practices is still a challenge.

This paper presents a work being carried out in order to extend the $\mathrm{SC}^{2}$ system (supply chain smart coordination) (Rabelo, 2004b). $\mathrm{SC}^{2}$ is a support system built up to help the VO coordinator to achieve a better management, with an emphasis on the information flow. The $\mathrm{SC}^{2}$ provides a set of approaches and techniques allowing managers to collect, analyze, and organize the information about multiple SCs and their individual chains in a VO context.

The developed functionalities allow VO members to access information available at different levels according to some visibility criteria - about the whole VO. These functionalities aid to minimize the system global costs and to improve the transparency of the joint business, besides motivating trust building and cooperation among partners (Rabelo, 2004b).

$\mathrm{SC}^{2}$ is a multi-agent system and it is composed by three classes of agents as shown in Figure 1: smart supervisor, chain supervisors, and supporting agents. The first one is the agent called Smart Agent, responsible for the global SC management. The second one has three instances: Production Agent, Sales Agent and Distribution Agent, responsible for dealing with the production, sales and distribution individual chains, respectively. The third one is represented by the XML Agent, which is responsible for dealing with the communication among the agents and with the "external environment".

Despite $\mathrm{SC}^{2}$ helps the managers to overcome the problems of dealing with a vast amount of information, it offers very few pro-active actions. Whenever the manager desires some information (s)he must consult reports and functionalities. So far, the system does not provide/suggest plans in order to improve management skills or to solve existing problems. Our aim is to expand the Smart Agent functionalities 
offering an integrated decision-making environment within which $\mathrm{AI}$ techniques are applied to support some levels of intelligence.

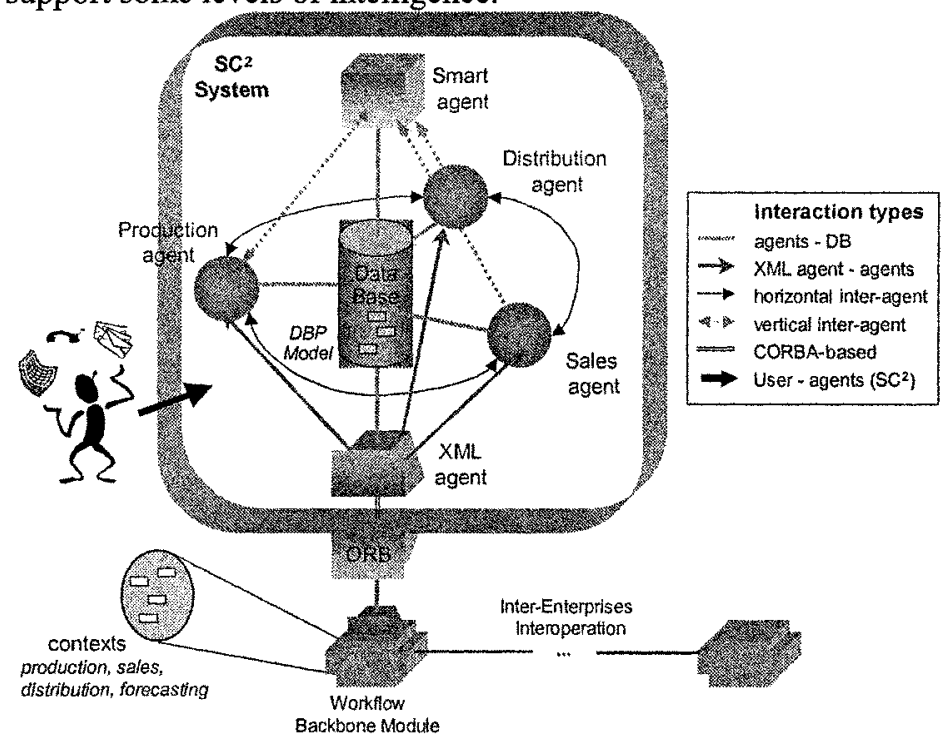

Figure 1 - The desktop SC ${ }^{2}$ Architecture

\section{IDENTIFYING RELATED TECHNOLOGIES}

According to Lavrac (2002) the recognizing of partners' expertise, tools and skills as marketable knowledge assets are factors in which the success of knowledge intensive services are critically depend. Problems like efficiently storing, updating, as well as sharing should be solved by the VO in order to promote and to transfer knowledge among its members. Appropriate management will lead to a quick recognition of business opportunities and timely responses. In this section some relevant technologies are depicted in order to help to understand their use in the proposed architecture. These technologies are:

- Data Mining is concerned with finding patterns in data which are interesting and valid (Fayyad, 1996).

- Text Mining is about looking for regularities, patterns or trends in natural language text, usually texts for particular purposes (Intelligent-Web, 2005).

- Decision Support is related to developing systems aimed at helping decision-makers to solve problems and make decisions (Mallach, 2000).

- Benchmarking is a continuous and systematic process to evaluate products, services and processes against competitors or renowned organizations considered world leaders in their field (Zairi \& Leonard, 1996).

- Machine Learning and the principle of learning that can be executed by computers and/or systems to improve their performance (Mitchell, 1997).

- Ontology formally represents domains of knowledge. Ontologies structures are formal explicit description of concepts in a domain of discourse, properties of 
each concept describing various features and attributes of the concept, and restrictions on slots (Konar, 1999).

- Knowledge base may be used in order to store the knowledge obtained through the VO life-cycle in a systematic way. It is an approach to structure and to codify what is known about an area/issue (Rezende, 2003).

\section{PROPOSED APPROACH}

The existing tools have not used techniques, like Artificial Intelligence (AI), as an alternative approach to improve system's efficiency. AI techniques may help solve unpredictable and unexpected problems suggesting ad hoc solutions. It means that the system will work either anticipating future actions to improve efficiency and to avoid errors, or working as an integrated environment where decisions are taken in intelligent way.

This paper proposes a system architecture based on computational decision support system called "Intelligent DSS" which is divided in two layers (Figure 2). The first layer is responsible for the intelligent processes and is also called back-end. The processes running on the back-end, the ones that will help on decision-making process, are considered intelligent because they are able to generate new knowledge and to reason about them as well as to use knowledge to solve problems (Rezende, 2003). The second layer is responsible for the smart processes (it is not intelligent indeed). The smart level is the interface where the manager will interact with the system; it is also called here front-end.

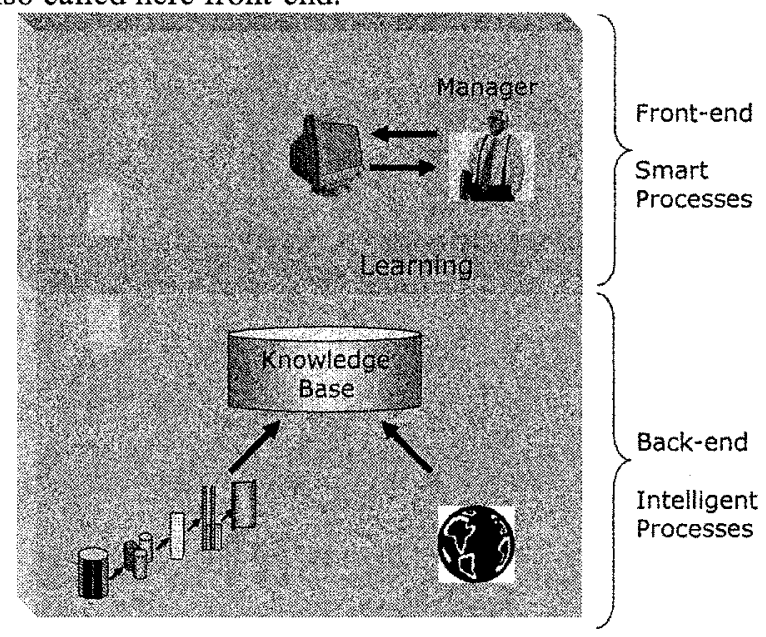

Figure 2 - Layers comprising the Intelligent DSS

The overall process is divided in two main steps, namely: off-line/intelligent processes, occurring in the back-end, and on-line/smart processes, occurring in the front-end. This division is necessary because before the manager starts to interact with the Intelligent DSS (on-line), the system must be fed with some knowledge, in a case-based learning way, (off-line) in order to attend the manager's requests.

The first step is executed off-line right after the VO had been created and had started its operation. The database is filled with current and historical data about 
each VO partner. The database is constantly updated as shown in Figure 3(a), despite this practice is not trivial, Rabelo et al., (2004a) has already proved the feasibility of this process. Once data is stored, it is necessary to extract relevant information in order to help on future decision-making task. The process is carried out by applying data and text mining techniques as shown in Figure 3(b).

Figure 3(c) illustrates the inclusion of pieces of information coming from the experience got from benchmarking techniques (external world). It is important to emphasize that when feeding information in the knowledge base an ontology is required. The cross-functional team ${ }^{3}$, on the other hand, is responsible to validate information and to include new information that seems to be relevant and/or to be useful for decision-making processes. In this approach the cross-functional team can also be called cross-organizational team because it includes people (experts) from each partner of the VO.

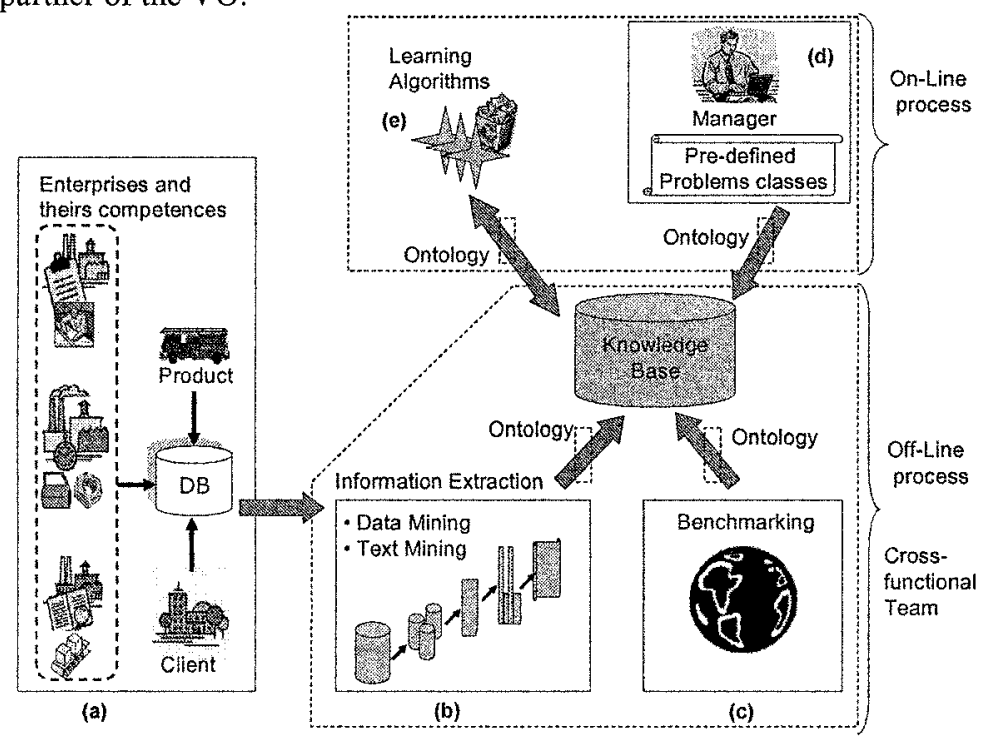

Figure 3 - Process to Obtain Information and Knowledge.

The second step, on-line, is started after all previous processes have been executed. At this time the manager interacts with the Intelligent DSS asking for advices in order to solve some problems (Figure $3(\mathrm{~d})$ ).

Reinforcement learning algorithms are applied on this task (Figure 3(e)) and are responsible for the "intelligent processes" of the system suggesting actions to the manager. These algorithms monitor all actions taken on the system and they are executed whenever a new piece of information is included in the knowledge base. The algorithms are able to classify the information, detect errors and delays, and previously alert the manager(s). The analysis is done based on past decisions in order to suggest the best actions to be taken on the decision process, by the manager. The manager still remains with a very important role which is the responsibility to

\footnotetext{
${ }^{3}$ The cross-functional team of an enterprise is composed by people with knowledge and experience in many areas. (engineers and managers with knowledge and ability to "shape" new knowledge, IT people to support system's infrastructure, and so on).
} 
validate the knowledge "created" by the system through the rank. The next section goes deeper in each phase where the steps are "instantiated" in a working scenario.

\subsection{Example}

In order to facilitate the comprehension, it is assumed that a VO is already created and is operating. The following illustrative situation is supposed: a large automobile enterprise wishes to contract a supplier to produce moulds and dies in order to build a new prototype in the Southern of Brazil. However, none mould and die industry placed in that area has enough resources to fulfill all requirements; rather, just part of them can be performed. The solution found by a group of five enterprises was to work collaboratively.

One enterprise (i.e. partner number 3) will take the role of a VO coordinator ${ }^{4}$ and will concentrate all information from the other four partners to manage the VO. The manager entrusted to manage the VO is called Robert Landfill. It is important to emphasize that the proposed tool should be used only by the VO coordinator, so that, other members do not interact directly with the system interface, they only provide data to a common VO database (Figure 3(a)).

While the VO is under operation, the Intelligent $D S S$ verifies that partner number 1 is delayed to deliver a mould (step 1, Figure 4). This delay is critical to the delivery time stipulated by the contractor. The problem is detected because the Intelligent DSS system has a specific module that continuously monitor each partner in the VO.

As soon as the Intelligent DSS detects the delay (comparing the actual data with the VO production planning), the system sends a standard alert message to Robert reporting the problem occurrence (step 2, Figure 4). At the same time the process to find possible solutions based on the knowledge stored on knowledge base is started.

The search for solutions is done comparing the detected problem in each class defined by the ontology against the knowledge base (step 3, Figure 4). Possible solutions are suggested to the manager through a report (step 4, Figure 4) i) to devote specialized employees to execute the task i.e. the system suggests to increase the working hours of a mechanical engineer to nineteen hours and of two material engineers to fourteen hours so that the production can be speed up (63\% efficient), or ii) to reschedule the distributed process i.e. to anticipate the beginning of task " $D$ " from partner 5 in three days and seven hours in order to compensate the delay on task " $\mathrm{B}$ " from partner 1 ( $71 \%$ efficient). The Intelligent DSS makes use of the knowledge obtained (and being acquired during the VO life-cycle) in order to help the decision making process.

These pieces of advices were obtained from the knowledge base according to the characteristics of the problem. So the Intelligent DSS concluded that this postpone may cause the bullwhip effect (Carlsson, 2002) resulting in nine days and two hours delay. Since those possible solutions were put in the knowledge base in the past in order to solve a similar situation, they were ranked by other managers with a "reward signal", which expressed how successful they were $(63 \%$ and $71 \%$ respectively). Now they are available and are suggested to Robert by the system. In

\footnotetext{
${ }^{4} \mathrm{VO}$ coordinator is the supervisor component related to VO activities.
} 
the meantime the system is waiting for Robert's analysis and reply (steps 5 and 6 , Figure 4).

Due to his experience, Robert knows that despite anticipating task " $D$ " may be more successful ( $71 \%$ than $63 \%$ ), partners rescheduling is a hard task, so he decides to accept the first option and calls the engineering sector delegating the task. Now Robert can come back to his normal job.

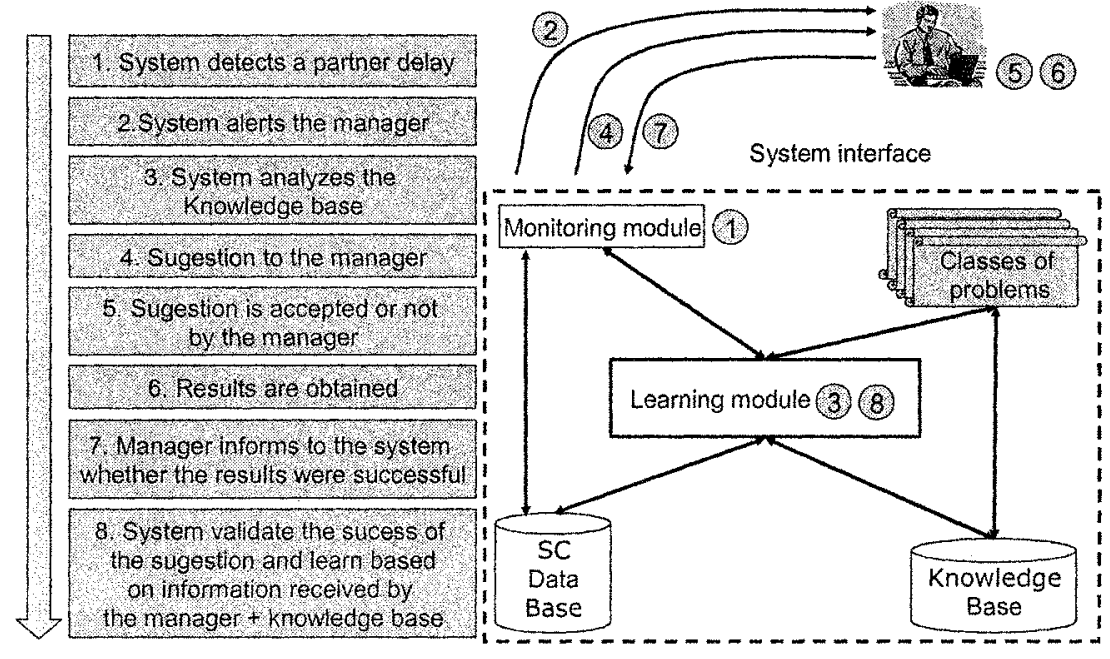

Figure 4 - Process of Decision-making and Learning

After task execution, Robert comes back to the Intelligent DSS and informs whether the results were successful or not (step 7, Figure 4). He accesses the system's options through the system interface and ranks how successful the options were. The rank ranges from -10 (bad suggestion) to 10 (terrific suggestion) where rank 0 means "I did not use it". Here the learning algorithms are triggered, where Robert gives the rewards related to each suggestion. The first option receives the value 9,0 , which means that it was a very good suggestion. The second option (rescheduling) receives value 0.0 , which means that the suggestion was not used.

The Intelligent DSS analyzes the rewards given by Robert, stores them to use as a validation parameter when a similar occurrence emerge, and inserts them in the knowledge base (step 8, Figure 4). It is not intended to provide optimal solutions using the Intelligent DSS, actually there is no accurate knowledge for one specific situation.

This is the so called "on-line" phase. However, the global process described above was possible because Robert used and has fed the system at least once and the cross-functional team has been worked in a good way in the "foundation" of the system, maintaining the system knowledge base properly updated. This knowledge preparation is the one carried out in the so called off-line phase on the system.

During the off-line phase, the Intelligent DSS's knowledge base must be fed with data in order to start effectively to work. The first action is to collect data from all partners to fill the VO database (Figure 3(a)).

After collecting data, the cross-functional team starts the process to extract relevant information from database. This is done applying data mining algorithms. 
These algorithms will help in the information discovery process. Usually this kind of information is undercover in a large amount of tables and registers, so managers do not have in mind its existence and its relationships, and consequently do not use it.

Now the cross-functional team executes the DM algorithms several times trying to find any association among data. For example, one of these associations is verified when there is a relationship among the amount of sales, the number of employees, and delayed products in the period from ten years to now (for each partner). The final analysis shows the enterprises divided into two groups and each group has their own characteristics. The former group containing three enterprises had the same variation in the sales during the period according to the market and does not registered serious problems with delays. However, the two other enterprises comprising the second group had an interesting behavior: sales were under $5 \%$ when the number of hired employees increased above $10 \%$.

By analyzing these data it is possible to conclude that the "phenomenon" occurs because hired employees do not had enough training to operate machines or they received insufficient trainings causing errors in the moulds during production and, consequently, delays in the delivery. At this point the cross-functional team has identified why delay has occurred.

Following the same line, text mining algorithms are also applied, but instead of searching for information in the VO database (like data mining), these algorithms look for information in internal documents and in web sites as an alternative source. Once again the cross-functional team specifies where the search must be done.

At this time, the cross-functional team realized that an important 50 pages document should also be comprised to complement the knowledge base. This document contains key indicators on how to improve efficiency on shop floor. Summaries are easier to use instead of the whole document when looking for possible solutions. i.e, results say that when a team which contains 10 employees working on the mould production is helped with 2 more senior engineers working 5 hours a day for 3 days, the final schedule are anticipated in 5 days and 7 hours.

Benchmarking rises up as an extra alternative to get information from outside and inside enterprises that are part of VO. This is done analyzing best practices. The cross-functional team evaluates actions taken during the bullwhip occurrence in well-known organizations, extracts how to proceed, and stores the information in the knowledge base. A best-practice says, for example, that enterprises should be transparent, strengthen strategic partnering arrangements with their distributors and customers, and should reduce order lead-times, besides to become a demand-driven enterprise through lean manufacturing.

All the processes executed by the cross-functional team are transparent to Robert. He does not to know how the Intelligent DSS works "downstairs". He just needs to know how to operate the system's interface.

It is important to emphasize that the more the Intelligent DSS is used, the richer are the knowledge to help the manager.

\section{CONCLUSIONS AND NEXT STEPS}

Collaborative Networks is an area that still requires much attention from the research community. This paper addressed the issue of managing virtual organizations as well as taking decision in a networked environment. 
It was observed that most of the problems both in traditional organizations and in recent VOs are solved in an ad hoc way without neither specific methodologies nor supporting tools. Yet, the available tools are not enough designed to help managers in smart decision-making. It was verified that although efforts are being carried out towards the identification of needs and generation of research recommendations on how VOs can be managed, they are not based on intelligent tools. Thus, the main goal of this work was to propose an integrated decision-making environment based on an ample set of combined Artificial Intelligence techniques. The decision support interface provides means for smart decisions, whereas the base of the system acts intelligently in the supplying of useful knowledge to the manager. The prototype implementation as well as its validation are the next steps of this research.

\subsection{Acknowledgments}

This work was partially supported by $\mathrm{CNPq}$ - The Brazilian Council for Research and Scientific Development (www.cnpq.br), and it has been developed in the scope of the Brazilian IFM project (www.ifm.org.br) and the European IST FP-6 IP ECOLEAD project (www.ecolead.org).

\section{REFERENCES}

1. Afsarmanesh, H.; Marik, V.; Camarinha-Matos, L. M.; 2004. Challenges of Collaborative Networks in Europe. In:. Collaborative Networked Organizations. 1st Edition. KAP. 2004. p. 77-90.

2. Bremer, C. F..; Mundim, A. P.; Michilini, F. V. S. et al.; 1999. A Brazilian Case of Coordination. In: PRO-VE'99. Proceedings p. 377-386.

3. Carlsson, C.; 2002. Decision Support in Virtual Organizations: The Case for Multi-Agent Support. In: Group Decision and Negotiation. 11: 185-221, 2002. Kluwer Academic Publishers. Netherlands.

4. Dobler, D. W.; Burt, D. N.; 1996. Purchasing and Supply Chain Management. Text and Cases. 6th Edition. McGraw-Hill. 1996.

5. ECOLEAD, 2005: European Collaborative Networked Organizations Leadership Initiative Technical Report: D32.1 deliverable of ECOLEAD.

6. Fayyad, U.; Piatetski-Shapiro, G.; Smyth, P.; 1996. From Data Mining to Knowledge Discovery in Databases. AAAIMIT Press, p.37-54, 1996.

7. Intelligent-Web, 2005. Web site accessed in January, 29, 2005. URL: (http://intelligent-web.org/wsm/).

8. Konar, Amit; 1999. Artificial Intelligence and Soft Computing: Behavioral and Cognitive Modeling of the Human Brain. CRC Press LLC.

9. Lavrac, N.; Urbancic, T.; Orel, A.; 2002. Virtual Organization for Data Mining and Decision Support: A Model for Networking Academia and Business. In: PRO-VE'02. Proceedings p. 389-396.

10. Mallach, E. G.; 2000. Decision Support Systems and Data Warehouse Systems. McGraw-Hill, 2000.

11. Mitchell, T.; 1997. Machine Learning. McGraw-Hill.

12. Rabelo, R. Je.; Baldo, F.; Tramontin Jr, R. J.; et al.; 2004a. Smart Configuration of Dynamic Virtual Enterprises. In: 5th IFIP Working Conference on Virtual Enterprises (Aug.: 23-26) Toulouse, França.

13. Rabelo, R. J.; Pereira-Klen, A. A.; Klen, E. R.; 2004b. Effective Management of Dynamic and Multiple Supply Chains. In: International Journal of Networking and Virtual Organizations. Vol. 2. n. 3. p. 193-208.

14. Rabelo, R. J.; Pereira-Klen, A. A.; 2002. Business Intelligence Support for Supply Chain Management. In: BASYS02 Proceedings, Cancun 2002 p.437-444.

15. Rezende, S. O.; Pugliesi, J. B; Melanda, E. A.; et al.; 2003. Mineração de Dados. In: Rezende, S. O.; Sistemas Inteligentes - Fundamentos e Aplicaçðes. 1st Edition. Barueri, SP: Manoele, 2003. p. 307-335.

16. Zairi, M.; Leonard, P.; 1996. Practical Benchmarking: The Complete Guide: London: Chapman \& Hall, 1996. 